\title{
Wave Interference for Pattern Description
}

\author{
Selen Atasoy ${ }^{1,2}$, Diana Mateus ${ }^{2}$, Andreas Georgiou ${ }^{1}$, \\ Nassir Navab ${ }^{2}$, and Guang-Zhong Yang ${ }^{1}$ \\ 1 Visual Information Processing Group, Imperial College London, UK \\ \{catasoy, a.georgiou, g.z.yang\}@imperial.ac.uk \\ 2 Computer Aided Medical Procedures and Augmented Reality, \\ Technical University of Munich, Germany \\ $\{$ atasoy, mateus, navab\}@in.tum.de
}

\begin{abstract}
This paper presents a novel compact description of a pattern based on the interference of circular waves. The proposed approach, called "interference description", leads to a representation of the pattern, where the spatial relations of its constituent parts are intrinsically taken into account. Due to the intrinsic characteristics of the interference phenomenon, this description includes more information than a simple sum of individual parts. Therefore it is suitable for representing the interrelations of different pattern components. We illustrate that the proposed description satisfies some of the key Gestalt properties of human perception such as invariance, emergence and reification, which are also desirable for efficient pattern description. We further present a method for matching the proposed interference descriptions of different patterns. In a series of experiments, we demonstrate the effectiveness of our description for several computer vision tasks such as pattern recognition, shape matching and retrieval.
\end{abstract}

\section{Introduction}

Many tasks in computer vision require describing a structure by the contextual relations of its consitituents. Recognition of patterns where the dominant structure is due to global layout rather than its individual texture elements such as the triangle images in Figure 1a-c and peace symbols in Figure 10a, patterns with missing contours such as the Kanizsa triangle in Figure 1d and Figure 5b and patterns with large homogeneous regions such as the shape images in Figure 9 , necessitate the description of contextual relations within a structure in an efficient yet distinct manner. The captured information has to be discriminative enough to distinguish between structures with small but contextually important differences and be robust enough to missing information. In this paper, we introduce a method based on wave interference that efficiently describes the contextual relations between the constituent parts of a structure.

Interference of several waves that originate from different parts of a structure leads to a new wave profile, namely the interference pattern (Figure 2). This interference profile is computed as the superposition of all constituent circular waves and has two relevant properties for describing a structure. Firstly, the 


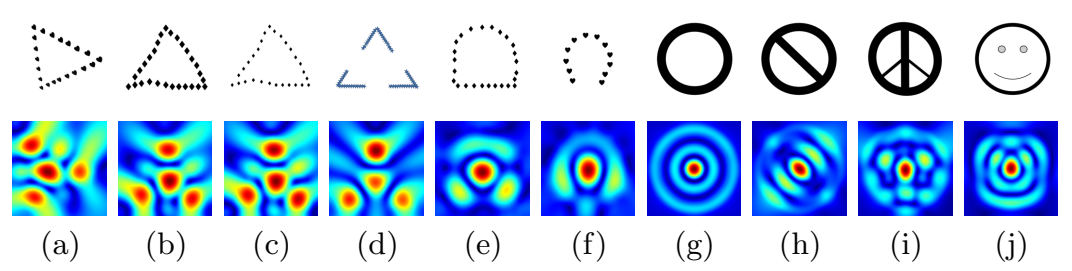

Fig. 1. Interference patterns created from input images (shown in the first row) with several geometric transformations (a-c) and missing contours (d) leads to the emergence of similar patterns (shown in the second row), whereas shapes with only very small variations yield very distinct interference patterns (e-j).

interference profile depends on the relative spatial arrangement of the sources that create the constituent waves. Secondly, it intrinsically captures all relationships of the constituent waves. Thus, interference phenomenon provides a natural mechanism to describe the structure of a pattern by representing the interrelations of its components. The resulting interference patterns are discriminative enough to separate different structures with only very small variation such as the examples shown in Figure 1e-j but not sensitive to a loss of information due to missing contours or to geometric transformations as illustrated in Figure 1a-d.

Recognition of patterns with missing contours is an important property of human perception which has been extensively studied by Gestalt psychology [1] This constructive property, known as "reification", is demonstrated in Figures 1d and $5 \mathrm{~b}$, where a triangle is perceived although it is not explicitly delineated. This is due to the spatial configuration of the constituent contours. Gestalt theory also points out two other relevant properties of human vision, i.e., invariance and emergence. "Invariance" analyses how objects are recognized invariant of their location, orientation and illumination. It is also an active area of research in computer vision. "Emergence" property states that a pattern is not recognized by first detecting its parts and combining them to a meaningful whole, but rather emerges as a complete object; i.e. "the whole is more than the sum of its parts".

In fact, the interference phenomenon intrinsically exhibits the emergence property. The interference pattern is produced by the superposition of two or more waves. This leads to the occurrence of new structures which are not present in any of the constituent waves nor in their simple sum but are created by the interrelations of the parts as shown in Figure 2.

The contribution of our work presented in this paper is twofold: firstly, we introduce the notion of wave interference for pattern description. To this end, we create interference patterns from an input image. This leads to an emergent description of the structure in the input pattern by representing the interrelations of its constituent parts. We demonstrate that our method, called "interference description" (ID), exhibits some of the key Gestalt properties including emergence, reification and invariance. Secondly, we use these interference patterns to 
create compact descriptor vectors and show in a series of experiments how the interference patterns can be applied to several computer vision tasks such as pattern recognition, shape matching and retrieval.

\section{Related Work}

In general, pattern description techniques can be analyzed in three main categories. Local methods extract and describe discriminative local information such as features and patches. The descriptors are then used for object matching or pattern recognition without using their contextual relations (See [2] and references inside). Global methods, such as descriptions based on the spectrum of Laplacian [3, 4] or finite element modal [5], capture distinctive information using the whole content of the pattern. In a third category, contextual methods, the local information is analyzed within the global context. To this end, relevant local information of the parts is combined with the contextual relationships between them. For instance, Sechtman and Irani create a descriptor using patches with a certain degree of self-similarities [6], Belongie et al. compute the shape context descriptors using distance histograms for each point. These descriptors are then usually combined with a suitable matching method such as star-graph [6] or hyper-graph matching [7], or a nearest neighbour matching after aligning two shapes [8].

One of the major challenges for modelling the contextual relations in the image domain is the computational complexity which increases exponentially with the order of relationships considered. Therefore, most methods include relations up to second order (pair-wise) [6,8] or third order [7]. Furthermore, for applications such as retrieval, shape comparison or pattern recognition, a more compact description of the whole structure is desired as the explicit computation of feature correspondences is actually not required.

Another form of global description methods, the frequency analysis techniques, on the other hand, allows the consideration of more complex relations of a pattern by encoding all global information. Fourier descriptors [9], for example, project the shape contour onto a set of orthogonal basis functions. Wavelet based methods such as [10] analyze the response to wave functions that are more localized in space and frequency. Spectral analysis using spherical harmonics [11] and eigenfunctions of the Laplace-Beltrami operator (vibration modes) has been successfully applied also for representation and matching of images [4] and 3D shapes [3].

In this paper, we introduce the notion of wave interference in order to create a compact pattern description. The proposed method intrinsically captures $n$ order relations ${ }^{3}$ without increasing the computational complexity. The novelty of our method lies in the fact that it analyses the interference, and thus the interaction, of circular waves over a range of frequencies, whereas other frequency techniques $([9-11,3-5])$ are based on the reponses at different frequencies that do not interact with each other.

\footnotetext{
${ }^{3} n$ is the number of all individual parts modelled as sources in our method.
} 


\section{Properties of Interference Description}

In this section we introduce and discuss some of the key properties of ID which are relevant for pattern description.

Modelling Contextual Relations: In ID, contextual relations emerge automatically due to the relation of individual circular waves. This has two important benefits: Firstly, it eliminates the need for explicit modelling of any order relations or for defining a set of rules. Secondly, all contextual relations of a pattern are considered without increasing the computational cost. The interference is computed by a simple addition of complex wave functions, where the computation of individual wave functions are independent. Therefore, including a new part to the pattern is simply done by adding its wave function to the sum, whereas in other contextual methods, all descriptors need to be re-computed in order to consider their relations to the newly added part. Furthermore, as the contextual relations are already included in the ID, comparison of descriptors for retrieval or recognition can be performed by simple nearest neighbor matching. This avoids the requirement of more sophisticated methods such as graph matching or non-linear optimization.

Specificity and Precision: In pattern description there exists a natural tradeoff between the specificity and precision of the method. ID allows to regulate this trade-off by the choice of the number of frequencies. A structure is described by a set of interference patterns computed over a range of frequencies (explained in Section 4.3), where the higher the frequency, the more specific the description becomes. Therefore the number of frequencies, which is the only parameter of the proposed method, allows adjusting the specificity of the description depending on the application.

Verification of Gestalt Properties: Gestalt psychology has played a crucial role in understanding human visual perception. It also has been an inspiring model for several computer vision applications such as analyzing shapes [12], learning object boundaries [13], defining mid-level image features [14] and modeling illusionary contour formation [15]. An extensive study of the Gestalt principles and their application in computer vision is presented in $[1,16]$. In this work, we point out the analogy between the interference phenomenon and three important Gestalt principles, namely emergence, reification and invariance. In Section 5.1 , we demonstrate that due to the particular nature of interference, our method intrinsically satisfies the mentioned Gestalt properties and eliminates the need for a set of defined rules or learning using a training dataset.

\section{Methods}

In this Section, we first introduce the background for waves and interference phenomenon. Then we explain our approach for pattern description, where an 


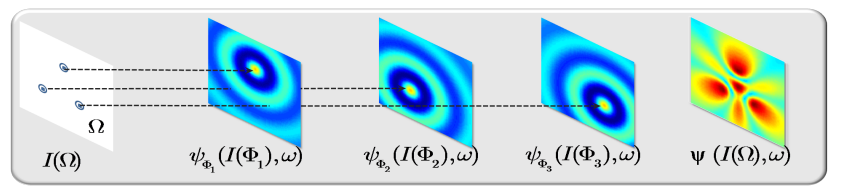

(a)

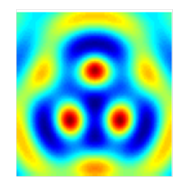

(b)

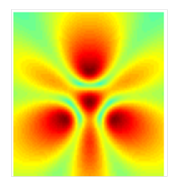

(c)

Fig. 2. a) Demonstration of the emergent nature of interference. Each source in the stimulus field creates a (attenuating) circular wave on the medium resulting in the final interference pattern. b) Sum of the individual wave patterns. c) Interference of individual wave patterns. The interference phenomena intrinsically exhibits the emergence property, as the whole (the interference pattern) shown in c) is different than the sum of its constituent wave profiles shown in b).

input pattern is first transformed into a field of sources; each creating a circular wave; and resulting jointly in an interference pattern. Finally, we propose a method to perform comparison of the created interference patterns.

\subsection{Background}

In its most basic form, a wave can be defined as a pattern (or distribution) of disturbance that propagates through a medium as time evolves. Harmonic waves are waves with the simplest wave profile, namely sine or cosine functions. Due to their special waveform they create wave patterns which are not only periodic in time but also in space. Thus, on a $2 \mathrm{D}$ medium, harmonic waves give rise to circular wave patterns such as the ones observed on a liquid surface when a particle in dropped into the liquid.

Using complex representation, a circular wave is described by the following wave function of any point $\mathbf{x}$ on the medium and a time instance $t$ :

$$
\psi_{\phi}\left(A_{0}, \omega ; \mathbf{x}, t\right)=A_{0} \cdot e^{i(\omega \| \mathbf{x}-\phi||)} \cdot e^{i \omega t},
$$

where $i$ is the imaginary unit, $\omega$ is the frequency of the wave and $\phi$ is the location of the source (stimulus). $A_{0}$ denotes the intensity of the source and $\|\mathbf{x}-\phi\|$ gives the distance of each point $\mathbf{x}$ on the medium to the location of the source.

As the time dependent term $e^{i \omega t}$ is not a function of space $(\mathbf{x})$, it does not affect the interference of the waves. Therefore, in the rest of the paper it will be taken as $t=0$. So, the instantaneous amplitude of the disturbance at each point on the medium is given by the real part of the wave function Real $\left[\psi_{\phi}\left(A_{0}, \omega ; \mathbf{x}\right)\right]$. For simplicity, we will denote waves with a fixed frequency $\omega$ and a fixed amplitude $A_{0}$ as $\psi_{\phi}(\mathbf{x}):=\psi_{\phi}\left(A_{0}, \omega ; \mathbf{x}\right)$.

As waves propagate outwards from the source, the amplitude of the wave will gradually decrease with the increased distance from the source due to friction. This effect, called attenuation, can be described by multiplying the amplitude of the wave with the attenuation profile $\sigma$.

When two or more waves $\psi_{\phi_{i}}$ are created on the same medium at the same time from several source locations $\phi_{i}$, the resultant disturbance $\Psi$ at any point $\mathbf{x}$ 
on the medium is the algebraic sum of the amplitudes of all constituents. Using complex represetation, the interference pattern $\Psi(\mathbf{x})$ is given by the amplitude of the sum of individual complex wave functions $\psi_{\phi_{i}}(\mathbf{x})$ :

$$
\Psi(\mathbf{x})=\left|\sum_{i=1}^{n} \psi_{\phi_{i}}(\mathbf{x})\right|,
$$

where $|\cdot|$ denotes the absolute value of the complex number. Note that the absolute value of the sum of a set of complex numbers is different than the sum of their real parts (See Figure 2). This is known as the superposition principle of the waves. The superposition of waves with fixed frequencies yields to a special phenomenon called interference. In this special case, if the waves reaching a point on the medium are in-phase (aligned in their ascent and descents), they will amplify each other's amplitudes (constructive interference); conversely if they are out-of-phase, they will diminish each other at that point (destructive interference). This results in a new wave profile called interference pattern.

Figure 2a illustrates the interference pattern created by the superposition of 3 waves with the same frequency. The interference pattern is computed by the absolute value of the sum of complex wave functions. The complex addition results in a waveform which is not present in any of the constituting waves nor in the simple sum of the contituent wave amplitudes (Figure $2 \mathrm{~b}$ ) but occurs due to their combination (Figure 2c). Therefore, interference property of waves provides an emergent mechanism for describing the relations between the sources and for propagating local information.

\subsection{Interference Description}

Using the introduced definitions in Section 4.1, our method formulates the contextual relations of the parts composing the image in an emergent manner.

Let $\Omega \subset \mathbb{R}^{2}$ be the medium on which the waves are created, where $\mathbf{x} \in \Omega$ denotes a point on $\Omega$. In practice the medium is described with a grid discretizing the space. The function $I: \Omega \rightarrow \mathbb{R}$, called stimulus field, assigns a source intensity $A_{0}=I(\phi)$ to the positions $\phi$ on the medium $\Omega$. This function contains the local information of the pattern which is propagated over the whole medium. Depending on the application, it can be chosen to be a particular cue defined on the image domain such as gradient magnitude, extracted edges or features.

Each value $I(\phi)$ of the stimulus field $I$ induces a circular wave $\psi_{\phi}(I(\phi), \omega ; \mathbf{x})$ on the medium. We use the frequency of the wave to describe the relation between the distribution of sources and the scale at which one wants to describe the pattern. This is discussed in detail in Section 4.3. The profile of the created circular wave is described as:

$$
\psi_{\phi}(I(\phi), \omega ; \mathbf{x})=I(\phi) \cdot e^{i(\omega(\| \mathbf{x}-\phi||)} \cdot \sigma,
$$

where $\sigma$ is the attenuation profile. We define the attenuation profile of the wave induced by the source $\phi$ with frequency $\omega$ as:

$$
\sigma(\phi, \omega ; \mathbf{x})=\omega \cdot e^{-\omega \cdot\|\mathbf{x}-\phi\|},
$$




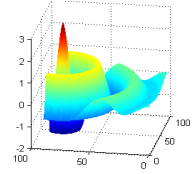

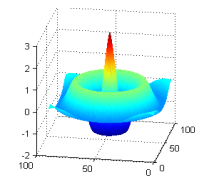

(a)
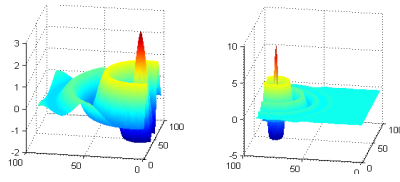

\section{for}

Fig. 3. Wave patterns for different source locations with the 3 rd frequency $(k=3)$ shown in a) and 8th frequency $(k=8)$ shown in b).

where $\|\mathbf{x}-\phi\|$ denotes the Euclidean distance between a point $\mathbf{x}$ and the source location $\phi$. This attenuation profile allows us to conserve the total energy of the waves while changing their frequency. Note that the attenuation profile is a function of the distance to the source $(\|\mathbf{x}-\phi\|)$ as well as the frequency $\omega$. The higher the frequency, the sharper is the decay of the attenuation profile. This yields to a localized effect for high frequencies and a more spread effect for low frequencies.

Once the location and intensities of the sources on the stimulus field are determined, the interference pattern $\Psi(I, \omega ; \mathbf{x})$ on the medium for a fixed frequency $\omega$ is computed simply by the superposition of all wave patterns:

$$
\Psi(I, \omega ; \mathbf{x})=\left|\sum_{\phi_{i} \in \Omega} \psi_{\phi_{i}}\left(I\left(\phi_{i}\right), \omega ; \mathbf{x}\right)\right| .
$$

\subsection{Multi-Frequency Analysis}

Computing the interference patterns for several frequencies allows for the analysis of the stimulus field at different scales. As discussed in Section 4.2, high frequency waves are more localized and propagate the content in a smaller neighbourhood, whereas low frequency waves are more spread over a larger area. Figure 3 illustrates the circular wave patterns for several frequencies and source locations.

Formally, we define the interference description (ID) $\Theta(I)$ of an input $I$ as the set of interference patterns $\Psi\left(I, \omega_{i}\right)$ for a range of frequencies $\left\{\omega_{1}, \omega_{2}, \ldots, \omega_{n}\right\}$ :

$$
\Theta(I)=\left\{\Psi\left(I, \omega_{1}\right), \Psi\left(I, \omega_{2}\right), \ldots, \Psi\left(I, \omega_{n}\right)\right\}
$$

with $\omega_{i}=\frac{2 \pi \cdot k_{i}}{P}$, where $k_{i} \in \mathbb{N}$ is the wave-number, i.e. the number of periods the wave has on the medium and $P$ is the length of the grid.

\subsection{Descriptor Comparison}

In this section, we present a method to compare two IDs, $\Theta\left(I_{1}\right)$ and $\Theta\left(I_{2}\right)$ in a rotation invariant manner. To this end, we first define a coherency measure $c$ of 


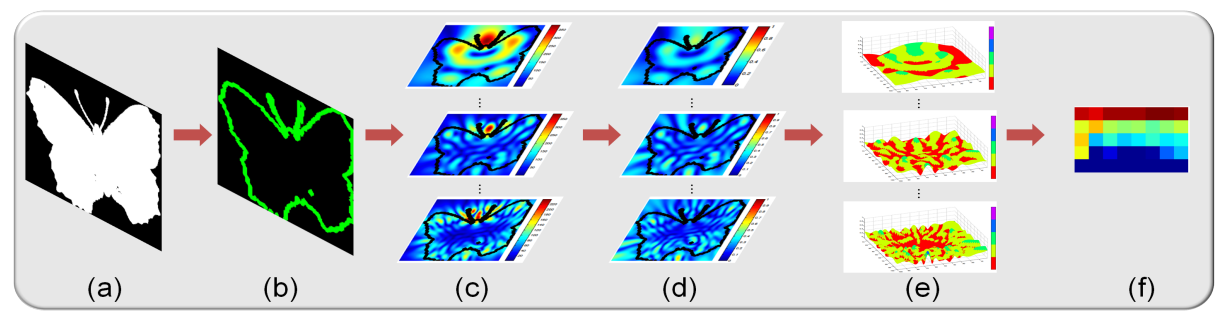

Fig. 4. Rotation invariant multi-frequency descriptor. a) Input image, b) sources located on the medium $\Omega$ as marked in green, c) interference patterns computed for a range of frequencies $\Theta(I), \mathrm{d}$ ) coherence measure computed on the interference patterns $c(\Theta(I))$, e) Levelsets $\left\{\gamma_{1}, \cdots, \gamma_{m}\right\}$ and f) the levelset histogram, where the columns correspond to descriptors at different frequencies.

an interference pattern $\Psi(\mathbf{x})$ :

$$
c(\Psi(\mathbf{x}))=\frac{|\Psi(\mathbf{x})|}{\sum_{\phi_{i} \in \Omega}\left|\psi_{\phi_{\mathbf{i}}}(\mathbf{x})\right|} .
$$

The coherency measures the power of the interference pattern compared to the sum of the powers of the constituent waves. As $\sum_{\phi_{i} \in \Omega}\left|\psi_{\phi_{\mathbf{i}}}(\mathbf{x})\right|$ provides the upper limit to the power of the interference amplitude $|\Psi(\mathbf{x})|$ for each location $\mathbf{x}$ on the medium, the coherency measure $c$ normalizes the values of the interference profile to the interval [0,1] (Figure 4c-d). This enables the comparison of different IDs independent of their absolute values and therefore makes the comparison independent of the number of sources.

We subdivide this coherency image $c(\Psi(\mathbf{x}))$ into $m+1$ levelsets

$$
\left\{\gamma_{0}, \gamma_{1}, \cdots, \gamma_{j}, \cdots, \gamma_{m}\right\} \quad \gamma_{j}=\{\mathbf{x} \mid c(\Psi(\mathbf{x}))=j / m\}
$$

and take the sum of the coherency values for each interval between the two consecutive levelsets. This yields an $m$-dimensional measure $h=\left[h 1, \cdots, h_{m}\right]^{\top}$, $h_{j}=\sum_{\gamma_{j-1} \leq x<\gamma_{j}} c(\Psi(\mathbf{x}))$ computed from an interference pattern $\Psi(\mathbf{x})$ (Figure 4e). Performing this levelset histogram calculation for the whole range of frequencies $n$ of an ID $(\Theta(I))$ results in a $m \times n$ dimensional measurement $h(\Theta(I))=\left[h\left(\Psi\left(I, \omega_{1}\right)\right), \cdots, h\left(\Psi\left(I, \omega_{n}\right)\right)\right]$ (Figure 4f). Quantifying the amount of coherency in each levelset enables a rotation-invariant description of the created interference patterns.

The distance between the two IDs $\Theta\left(I_{1}\right)$ and $\Theta\left(I_{2}\right)$ is then simply computed as the cosine measure between the levelset histograms:

$$
\operatorname{dist}\left(I_{1}, I_{2}\right)=\operatorname{acos}\left(\frac{h\left(\Theta\left(I_{1}\right)\right)^{\top} \cdot h\left(\Theta\left(I_{2}\right)\right)}{\left\|h\left(\Theta\left(I_{1}\right)\right)\right\| \cdot\left\|h\left(\Theta\left(I_{2}\right)\right)\right\|}\right) .
$$

This dissimilarity, measures the distance between the two patterns in a rotation invariant manner (due to the levelset histograms) while taking the contextual relations between the constituent parts (due to the interference pattern) for each pattern. 


\section{$5 \quad$ Experiments and Results}

Firstly, we demonstrate that the ID exhibits the Gestalt properties discussed in Section 2. Then, we show its application for shape matching and retrieval and pattern recognition.

For our experiments, we first create a grid (medium $\Omega:[-\pi \times \pi] \times[-\pi \times \pi]$ ) and map the locations of the stimulus field to the sources on the medium. Each source creates a circular wave pattern propagating outwards from this source location. Also, we define the stimulus field to be the gradient magnitude of the input image. Note that the use of more sophisticated feature extraction methods such as phase congruency [17] can further improve the performance. The choice of gradient magnitude is only due to its computational simplicity. The strength of the disturbance $\left(A_{0}\right)$ at a location $\mathbf{x}$ on the medium is set to be the gradient magnitude at that location.

\subsection{Verification of Gestalt Properties}

In this Section we demonstrate how the proposed description exhibits the Gestalt properties emergence, reification and invariance.

Emergence: The emergence property states that the whole (structure) is more than the sum of its parts. This is due to the interrelations of the constituents. In ID, the whole (i.e. interference pattern as shown in Figure 2c) is given by the amplitude of the complex interference field (sum of the complex wave functions). This interference pattern is different from the sum of the amplitudes of the consituent waves (Figure $2 \mathrm{~b}$ ). This is due to the constructive and destructive interference effect (as explained in Section 4.1) and can be seen mathematically as:

$$
\Psi(\mathbf{x})=\left|\sum_{i=1}^{n} \psi_{\phi_{i}}(\mathbf{x})\right| \neq \sum_{i=1}^{n} \operatorname{Real}\left[\psi_{\phi_{i}}(\mathbf{x})\right]=\sum_{i=1}^{n} \psi_{\phi_{i}}(\mathbf{x})
$$

where the left side of the inequality defines the interference pattern and the right side gives the sum of the amplitudes of its constituent waves. The difference between the whole and the sum of its parts leads to the emergence of a new profile which is not present in any of the constituent circular waves nor in their simple sum but occurs due to their interrelations.

Reification: Reification is the constructive property seen in human perception $[1,18]$. In Figures $5 \mathrm{~b}$ and $\mathrm{f}$, a triangle and a square are perceived, although they are not explicitly delineated. This is due to the particular spatial arrangement of the packman shapes. ID results in emergence of the same interference patterns in the location where a triangle is perceived in Figures 5a and b and where a square is perceived in Figures 5c and d. Therefore, ID also allows to recognize patterns with missing contours such as the triangle image in Figure 1d. 


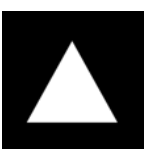

(a)

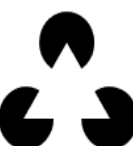

(b)

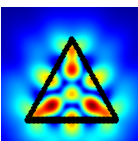

(c)

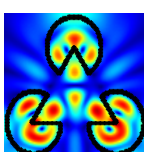

(d)

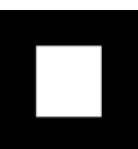

(e)

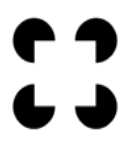

(f)

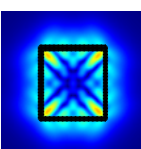

(g)

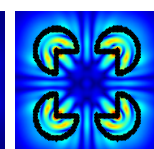

(h)

Fig. 5. Reification property seen in wave interference. Similar interference patterns emerge for a complete triangle a) and a Kanizsa triangle image b) or a complete square e) and a Kanizsa square image f). c) and d) demonstrate the interference patterns for the 4 th frequency $(k=4)$ for the triangle and Kanizsa triangle, respectively. The source locations are illustrated in black crosses. g) and h) show the interference patterns of images in e) and $\mathrm{f}$ ) for the 8 th frequency $(k=8)$.

Invariance: The invariance of ID to scale change is achieved due to the mapping of the stimulus field onto the same medium (discretized grid of fixed size). This creates sources along contours of the same size, however, the image with a smaller scale results in less number of sources sampled along the contour. The structure of the created interference patterns are the same as shown in Figures 6a,b. As the proposed comparison method in Section 4.4 is independent of the number of sources, the two IDs result in very similar levelset histograms as shown in Figure $6 a, b$.

We further demonstrate the invariance of the ID for rotation, affine and perspective transformations, as well as deformation. To this end, we simulate a binary triangle image and apply the corresponding transformations. Figure 6c-g displays for each transformation the input images, interference patterns for the same example frequency and the corresponding levelset histograms computed as described in Section 4.4. Note the similarity of the IDs and the levelset histograms despite the large geometric transformations between the images.

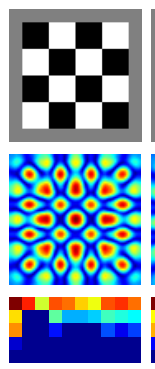

(a)
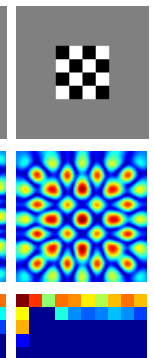

(b)
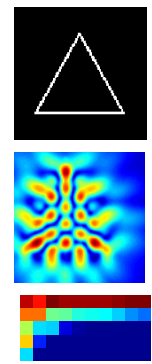

(c)

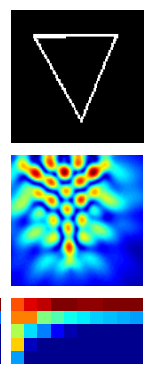

(d)

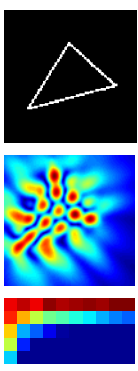

(e)

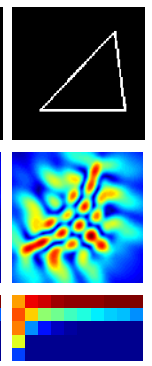

(f)

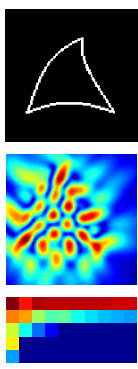

(g)

Fig. 6. Invariance of ID for scale change $(a, b)$, rotation $(c, d)$, affine transformation $(\mathrm{c}, \mathrm{e})$, perspective transformation $(\mathrm{c}, \mathrm{f})$ and deformation $(\mathrm{c}, \mathrm{g})$. First row shows the input patterns, second row illustrates interference pattern for the 6 th frequency $k=6$ and last row demonstrates the levelset histograms computed from the IDs $h(\Theta(I))$. 


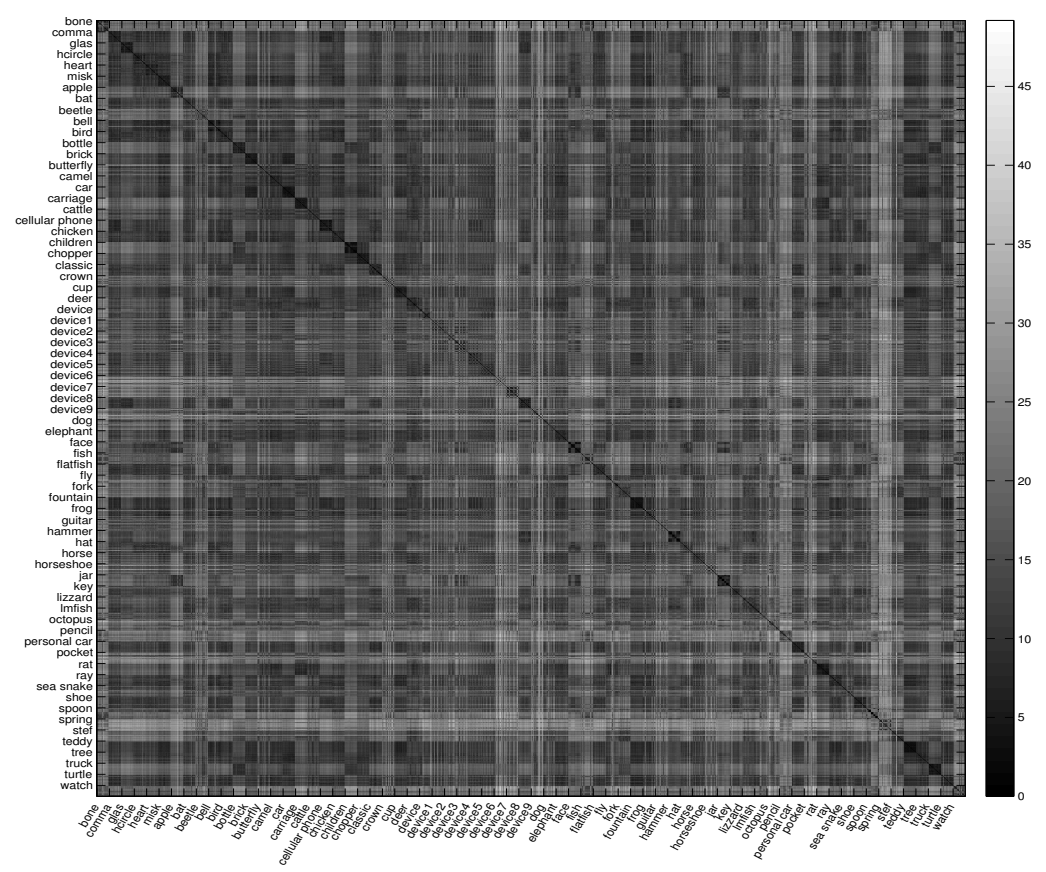

Fig. 7. The confusion matrix of ID matching as applied on the MPEG7 database.

\subsection{Application to Shape Matching and Retrieval}

In this experiment, we demonstrate the use of IDs for shape matching. We apply the presented description approach to match objects from the contour images of the MPEG7 CE shape database containing 1400 images with 70 different categories. For each image, we first compute the interference patterns for 10 frequencies. Then we compute the similarity of each shape image against all other images in the database by comparing the IDs as described in Section 4.4. Figure 7 demonstrates the performance of ID matching as a confusion matrix computed from the complete MPEG7 database.

After matching the shape images, we assign the best matched category to each image for retrieval considering the 20 minimum distances. Figure 8 displays the recall and precision over the 70 categories. Figure 9a demonstrates 3 example shapes; chopper, guitar, and spoon, where chopper leads to high recall and precision in the retrieval because of its characteristic structure. Due to their high similarity, guitar is confused with spoon in the final category retrieval. However, as demonstrated in Figure 9a, the interference patterns of the two shapes still show some differences despite the significant similarity between them. The confusion is caused due to the discretized levelset histogramming as illustrated in Figure 9a last row. In order to evaluate the inter-category distances, we embed the levelset histogram vectors (Section 4.4) linearly in to the 2D space using the 

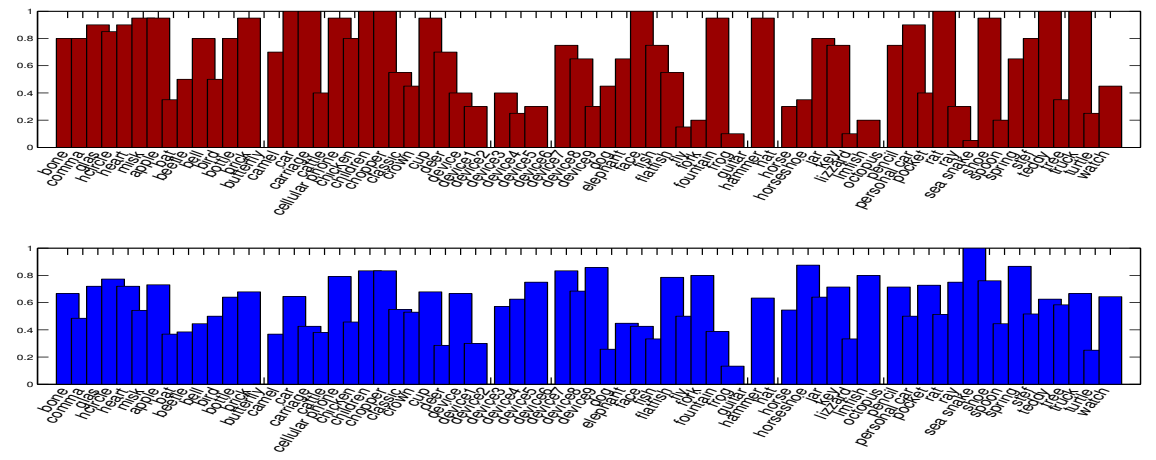

Fig. 8. a) Recall and b) precision values for shape category retrieval of IDs when applied to all categories in the MPEG7 shape database.

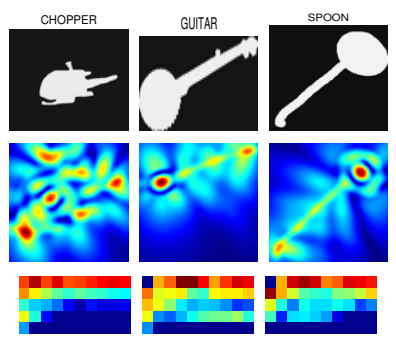

(a)

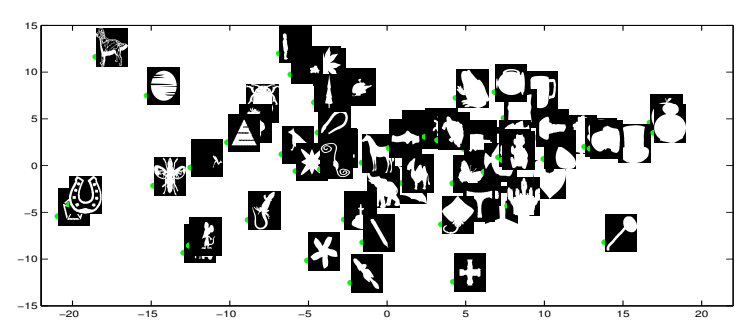

(b)

Fig. 9. a) First row: input images for 3 example shapes. Second row: the corresponding interference patterns for the 5 th frequency $(k=5)$. Third row: Levelset histograms computed with 5 levelset intervals for 10 frequencies. b) 2D MDS plot showing a representative for each category shape. Even in $2 \mathrm{D}$ representation similar categories are closely located whereas distinct categories are more separated.

classical multi-dimensional scaling (MDS). Figure 9b shows the 2D MDS plot of the representatives of each category. The MDS plot demonstrates how similar shape categories locate more closely whereas more distinct structures are more separated.

\subsection{Application to Pattern Recognition}

In this experiment we demonstrate that ID can be used to recognize patterns created by different texture elements. Therefore, we use images with large inter-class variability in the representation where in each image the pattern is generated with different texture elements. Although the details of the patterns are different, they all share the same global layout. This global structure is enhanced in the low frequency interference patterns. Therefore we create IDs $\Theta(I)$ for $k=\{1, \cdots, 4\}$. For a quantitative evaluation we compare the levelset histograms created from 

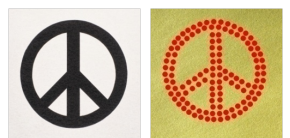

(a)
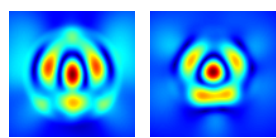

(b)

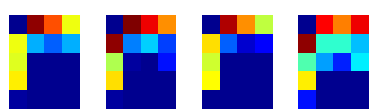

(c) ID
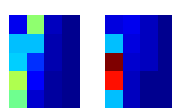

(d) Intensities
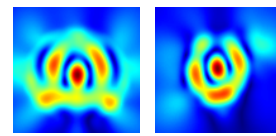

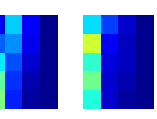

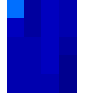

(e) Gradient

Fig. 10. Application of ID for pattern recognition. a) Input images containing the same global layout created with different texture elements. b) IDs at the frequency $(k=3)$ of the images a-d). c) Levelset histograms as compared to histogram of intensities d) and gradients e). For visualization the histograms are reshaped to $5 \times 4$ matrices.

the IDs to the histograms of gradients and of the intensities. Figure 10b illustrates IDs created from the images shown in Figure 10a. Figure 10c demonstrates their matching levelset histograms. IDs lead to similar but distinctive patterns, whereas the gradients or intensities lead to less similar histograms (Figure 10d,e) that also lack the discriminative power. The noisy background in the 4 . image in Figure 10a results in high gradient values in the background. This is also reflected in its effect on the histogram of gradients (4. image in Figure 10e). Naturally, this includes several sources due to the background, as the stimulus field $I(\phi)$ is defined to be the gradient magnitude. However, as shown in Figure 10b, ID tolerates a certain degree of background noise.

\section{Discussion and Conclusions}

In this work, we have introduced a method for pattern description that is based on wave interference. Due to the characteistics of interference phenomenon, our method intrinsically accounts for the contextual relations between the parts of a pattern. This eliminates the need for defining a set of rules or for any prior learning. Furthermore, the particular mathematical formulations of the presented method allow for the computation of higher order relations in an efficient manner, i.e. a simple complex addition. In a series of experiments, we have showed that the proposed description is in agreement with three key Gestalt properties relevant for pattern description. Future work will direct towards extending this approach for describing a whole scene including different objects and patterns. This requires creating an interference description for each object and then describing the scene hierarchically at different scales. In this work, we particularly pointed out the analogies of the wave interference phenomenon and some desired properties of pattern description and demonstrated its efficient application for pattern recognition, shape matching and retrieval. 
Acknowledgements. This research was supported by the Graduate School of Information Science in Health (GSISH) and the TUM Graduate School. The authors would like to thank Wellcome Trust and EPSRC for funding The Centre of Excellence in Medical Engineering and Tobias Wood and Ozan Erdogan for valuable discussions.

\section{References}

1. Koffka, K.: Principles of Gestalt psychology. Routledge (1999)

2. Lowe, D.: Object recognition from local scale-invariant features. International Conference on Computer Vision, ICCV 2 (1999) 1150-1157

3. Levy, B.: Laplace-beltrami eigenfunctions: Towards an algorithm that understand s geometry. IEEE International Conference on Shape Modeling and Applications (2006) 13-25

4. Peinecke, N., Wolter, F.E., Reuter, M.: Laplace spectra as fingerprints for image recognition. Computer-Aided Design 39 (2007) 460-476

5. Sclaroff, S., Pentland, A.: Modal matching for correspondence and recognition. IEEE Trans. on Pattern Analysis and Machine Intelligence 17 (1995) 545-561

6. Shechtman, E., Irani, M.: Matching local self-similarities across images and videos. IEEE Conference on Computer Vision and Pattern Recognition, CVPR (2007) $511-518$

7. Zass, R., Shashua, A.: Probabilistic graph and hypergraph matching. IEEE Conference on Computer Vision and Pattern Recognition, CVPR (2008) 1-8

8. Belongie, S., Malik, J., Puzicha, J.: Shape matching and object recognition using shape contexts. IEEE Trans. on Pattern Analysis and Machine Intelligence (2002) 509-522

9. Zhang, D., Lu, G.: A comparative study of Fourier descriptors for shape representation and retrieval. Asian Conference on Computer Vision, ACCV (2002)

10. Kuthirummal, S., Jawahar, C., Narayanan, P.: Planar shape recognition across multiple views. International Conference on Pattern Recognition 16 (2002) 456459

11. Kazhdan, M., Funkhouser, T., Rusinkiewicz, S.: Rotation invariant spherical harmonic representation of 3d shape descriptors. Eurographics/ACM SIGGRAPH Symposium on Geometry Processing (2003) 156-164

12. Loncaric, S.: A survey of shape analysis techniques. Pattern Recognition 31 (1998) 983-1001

13. Dollar, P., Tu, Z., Belongie, S.: Supervised learning of edges and object boundaries. IEEE Conference on Computer Vision and Pattern Recognition, CVPR 2 (2006)

14. Bileschi, S., Wolf, L.: Image representations beyond histograms of gradients: The role of Gestalt descriptors. IEEE Conference on Computer Vision and Pattern Recognition, CVPR (2007) 1-8

15. Williams, L., Jacobs, D.: Stochastic completion fields: A neural model of illusory contour shape and salience. Neural Computation 9 (1997) 837-858

16. Desolneux, A., Moisan, L., Morel, J.: From gestalt theory to image analysis: a probabilistic approach. Springer Verlag (2007)

17. Kovesi, P.: Image features from phase congruency. Videre: Journal of Computer Vision Research 1 (1999) 1-26

18. Carman, G., Welch, L.: Three-dimensional illusory contours and surfaces. Nature (1992) 